\title{
Benefícios da equoterapia em crianças com paralisia cerebral: revisão integrativa
}

\author{
Horse therapy benefits in children with cerebral palsy: integrative review \\ Beneficios de la terapia con caballos en niños con paralisis cerebral: revisión integrativa
}

Recebido: 30/01/2021 | Revisado: 05/02/2021 | Aceito: 09/02/2021 | Publicado: 16/02/2021

\author{
Matheus Braga de Lima \\ ORCID: https://orcid.org/0000-0003-4064-2254 \\ Universidade Federal do Rio Grande do Sul, Brasil \\ E-mail: mbl.matheus@hotmail.com \\ Simone Algeri \\ ORCID: https://orcid.org/0000-0002-3152-0944 \\ Universidade Federal do Rio Grande do Sul, Brasil \\ E-mail: simone.salgeri@gmail.com \\ Priscila Arruda da Silva \\ ORCID: https://orcid.org/0000-0002-5359-8646 \\ Universidade Federal do Rio Grande, Brasil \\ E-mail:patitaarruda@yahoo.com.br \\ Michele Peixoto da Silva \\ ORCID: https://orcid.org/0000-0002-4350-0588 \\ Prefeitura Municipal do Rio Grande, Brasil \\ E-mail:chele.p@hotmail.com \\ Naila Bragança Carvalho de Oliveira \\ ORCID: https://orcid.org/0000-0002-9946-3691 \\ Faculdade Redentor do Rio de Janeiro, Brasil \\ E-mail: nailab1@hotmail.com \\ Ana Cristina Wesner Viana \\ ORCID: https://orcid.org/0000-0002-0290-8288 \\ Universidade Federal de Ciências da saúde de Porto Alegre, Brasil \\ E-mail: wesner.viana@gmail.com
}

\begin{abstract}
Resumo
Analisar, na literatura científica, os benefícios da equoterapia em crianças com paralisia cerebral. Trata-se de uma revisão integrativa da literatura, com buscas nas bases de dados SciELO, LILACS e PubMed. Utilizou-se os descritores em ciências da saúde (DeCS), sendo eles: terapia assistida por cavalos, paralisia cerebral, criança, nos idiomas português, inglês e espanhol. Foram selecionados artigos completos de 2005 a 2020. A amostra foi composta por 11 artigos completos, organizados e caracterizados, de acordo com autor, título, ano, país do estudo, delineamento e os benefícios da terapêutica. A equoterapia, possibilita a melhora significativa da função motora, assim como na qualidade de vida, ao promover o autocuidado, melhora na autoestima, além da interação social. O estudo oferece importantes contribuições aos profissionais de diversas áreas do conhecimento, quanto aos benefícios da equoterapia, no desenvolvimento biopsicossocial da criança com paralisia cerebral.
\end{abstract}

Palavras-chave: Terapia assistida por cavalos; Equoterapia; Hipoterapia; Paralisia cerebral; Criança.

\begin{abstract}
To analyze, in the scientific literature, the benefits of hippotherapy in children with cerebral palsy. Method: This is an integrative literature review, searching the SciELO, LILACS and PubMed databases. In health sciences (DeCS) were used: horse-assisted therapy, cerebral palsy, child, in Portuguese, English and Spanish. Complete articles from 2005 to 2020 were selected. The sample consisted of 11 complete articles, organized and characterized according to author, title, year, country of study, design and benefits of therapy. Equine therapy enables the significant improvement of motor function, as well as quality of life, by promoting self-care, improvement in self-esteem, and social interaction. The study offers important contributions to professionals from various areas of knowledge regarding the benefits of hippotherapy in the biopsychosocial development of children with cerebral palsy.
\end{abstract}

Keywords: Equine-assisted therapy; Horseback riding therapy; Hippotherapy; Cerebral palsy; Child.

\section{Resumen}

Analizar, en la literatura científica, los beneficios de la hipoterapia en niños con parálisis cerebral. Esta es una revisión bibliográfica integradora que busca en las bases de datos SciELO, LILACS y PubMed. Se utilizaron los descriptores en ciencias de la salud (DeCS): terapia asistida por caballos, parálisis cerebral, infantil, en portugués, inglés y español. Se seleccionaron artículos completos de 2005 a 2020. La muestra consistió en 11 artículos completos, organizados y 
caracterizados según el autor, título, año, país de estudio, diseño y beneficios de la terapia. La terapia equina permite la mejora significativa de la función motora, así como la calidad de vida, al promover el autocuidado, la mejora de la autoestima y la interacción social. El estudio ofrece importantes contribuciones a profesionales de diversas áreas del conocimiento sobre los beneficios de la hipoterapia en el desarrollo biopsicosocial de niños con parálisis cerebral.

Palabras clave: Terapía asistida por caballos; Equino terapia; Hipoterapia; Parálisis cerebral; Niño.

\section{Introdução}

A paralisia cerebral são desordens motoras e posturais que resultam em alterações no sistema nervoso central (ABPC, 2012) a qual interfere nos movimentos e postura das crianças. Essas alterações variam de criança para criança, de acordo com a região lesada, porém as alterações mais encontradas são a espasticidade, hipotonia, comprometimento cognitivo, sensorial, visual, auditivo e o equilíbrio (Graham et al, 2016; Hidecker et al, 2018)

Como consequência, há dificuldades na execução de suas atividades de vida diária e podem limitar o convívio em ambientes como a escola, que muitas vezes não estão adaptadas para crianças com dificuldades de locomoção. Além destas alterações, as crianças com PC podem apresentar consequências secundárias, tais como dificuldade de aprendizagem, distúrbios na fala, problemas ortopédicos e em casos mais graves a epilepsia (Meincke et al, 2018; Park et al, 2016; ShikakoThomas et al, 2013).

A equoterapia, é um exemplo de tratamento que auxilia na recuperação desses pacientes. Consiste na utilização cavalo como forma de terapia, apresentando evidências de resultados benéficos em aspectos como concentração, socialização, organização do esquema corporal, estimulação do equilíbrio, regulação do tônus muscular e na autoestima dos pacientes (Prieto et al, 2018).

Aprovado recentemente, o projeto de Lei n. 13.830 de 13 de maio de 2019, regulamenta a equoterapia como um método de tratamento e reabilitação utilizado nas áreas de saúde, educação e equitação (Brasil, 2019). De acordo com a lei, a atividade passa a ser exercida por uma equipe multiprofissional, composta por médico, médico veterinário, fisioterapeuta, psicólogo e profissional de equitação, podendo integrar outros profissionais como terapeuta ocupacional, pedagogo, fonoaudiólogo, e professores de educação física, desde que possua curso específico de equoterapia (Brasil, 2019).

Destarte, considera-se a equoterapia como um campo novo no âmbito da saúde e educação, e que vem contribuindo para a melhora da qualidade de vida de crianças e adultos com limitações físicas e psíquica decorrentes da paralisia cerebral.

Por essa razão faz-se necessário conhecer como a equoterapia tem beneficiado crianças com paralisia cerebral. Considerada como um método de tratamento alternativo e complementar ao tradicional, voltada ao desenvolvimento biopsicossocial da pessoa com deficiência, este estudo surge com a finalidade de conhecer, a partir literatura científica os benefícios da equoterapia em crianças com paralisia cerebral.

Assim, o objetivo do estudo é investigar e analisar, na literatura científica, os benefícios da equoterapia em crianças com paralisia cerebral.

\section{Metodologia}

Trata-se de uma revisão integrativa da literatura, que tem como base, evidências científicas encontradas em estudos realizados em países desenvolvidos ou em desenvolvimento, os quais seus resultados podem ser aplicados na prática clínica. A busca por estudos com o mesmo tema e agrupados em quadros sinópticos, tem o objetivo sintetizar, concluindo de forma abrangente o fenômeno estudado (Cooper, 1982).

A pesquisa foi realizada durante os meses de novembro e dezembro de 2020, a partir de seis etapas metodológicas da revisão integrativa. A primeira etapa foi norteada pela seguinte questão de pesquisa: quais os benefícios da equoterapia em crianças com paralisia cerebral? Após a formulação da pergunta, definiu-se as palavras-chave, de acordo com os descritores em 
ciências da saúde (DeCS), sendo eles: terapia assistida por cavalos, equoterapia, hipoterapia, paralisia cerebral, criança, nos idiomas português, inglês e espanhol.

$\mathrm{Na}$ segunda etapa foram selecionadas as bases de dados e os critérios de inclusão e de exclusão do estudo. Foram incluídos no estudo, todos os artigos primários, publicados a partir do ano de 2005, até dezembro de 2020. O recorte temporal foi definido em função do período da criação do Projeto de Lei nº 5499/05 (Brasil, 2005) aprovado pela câmara dos deputados em 2005. Definiu-se como critérios de exclusão, artigos sem acesso livre on-line ao resumo, que não abordassem a temática de estudo, estudos de revisão, meta-análise, teses de doutorado, dissertações de mestrado, monografias, documentos e anais de eventos. A pesquisa foi realizada em base de dados, informatizadas, SciELO (Scientific Electronic Library Online), PubMed e LILACS (Literatura Latino-Americana e do Caribe em Ciências da Saúde). A seleção foi realizada a partir da leitura dos títulos, posteriormente os resumos e por fim os artigos na integra. A Figura 1 apresenta o fluxograma da busca nas bases de dados.

Para a terceira etapa foi elaborado um instrumento para a organização e caracterização dos resultados encontrados no quadro 1, que contemplou: autor, título, ano, país do estudo, delineamento e desfecho (benefícios da equoterapia).

$\mathrm{Na}$ quarta etapa foi realizada a análise dos resultados previamente selecionados e aplicados os critérios de inclusão e exclusão. A busca resultou em onze estudos, nove da base de dados da PubMed; nenhum da SciELO e dois da LILACS. Os onze artigos foram analisados na íntegra, buscando responder à questão norteadora do estudo. Com base nos artigos analisados, foi possível apontar os benefícios da utilização da terapia em cavalos no cuidado em crianças com paralisia cerebral para sua melhor qualidade de vida.

\section{Resultados e Discussão}

Na busca inicial foram identificadas 42 publicações. Após a exclusão de duplicatas e avaliação de títulos e resumos ( $\mathrm{n}=16)$ e artigos na integra $(\mathrm{n}=15)$, a amostra final foi de 11 artigos. A maioria dos artigos excluídos, eram estudos secundários e realizados por autores Brasileiros. Foram excluídos ainda, estudos que utilizavam simulares e robôs.

Figura 1: Fluxograma da sistematização da busca nas bases de dados PubMed, LILACS e SciELO, Rio Grande, Rio Grande do Sul, Brasil, 2020.

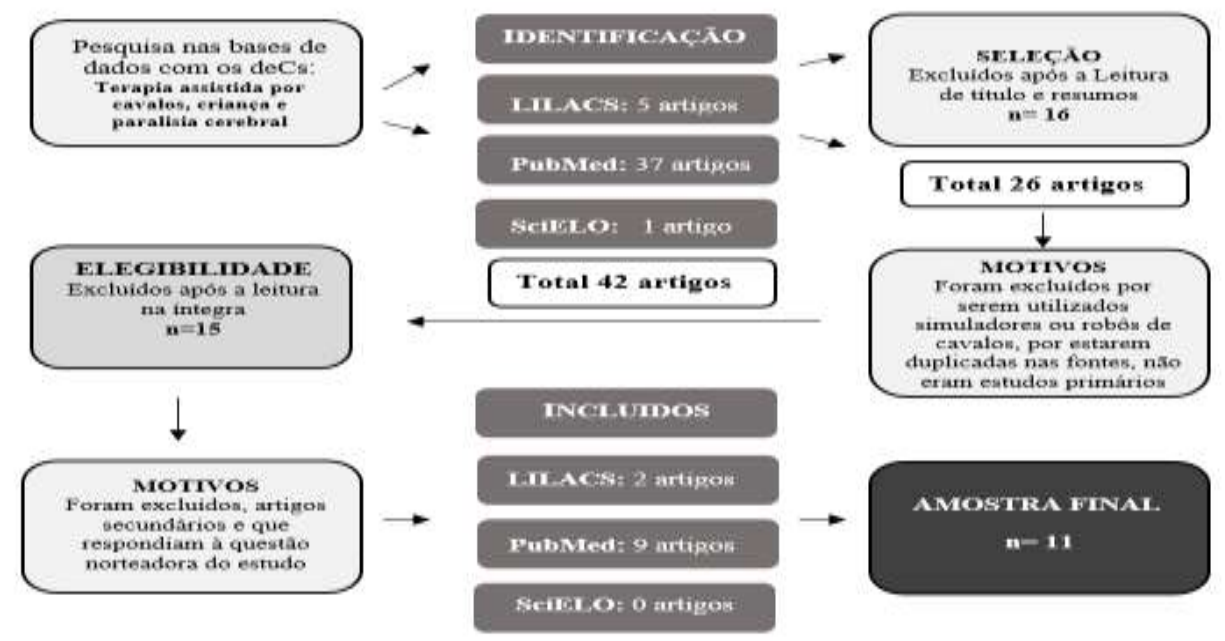

Fonte: Autores. 


\section{Caracterização da Amostra}

A amostra foi constituída por onze estudos, realizados em diferentes países seguindo as seguintes distribuições: 2 estudos na América do Sul (Brasil); 2 estudos na América do Norte (Estados Unidos); 5 estudos na Ásia (Japão, Coréia, Taiwan), 2 estudos na Europa (Polônia e Espanha). Quanto ao período, 2011 e 2016 foram publicados dois estudos respectivamente. No Quadro 1 está a síntese dos artigos selecionados para o presente estudo, apresentados em ordem cronológica crescente:

Quadro 1 - Caracterização dos estudos incluídos na pesquisa.

\begin{tabular}{|c|c|c|c|c|}
\hline Autor (ano) & Título & País & Delineamento & Benefícios da equoterapia \\
\hline $\begin{array}{l}\text { Silva, } \\
\text { Pietro et al } \\
(2019)\end{array}$ & $\begin{array}{l}\text { Efeitos da equoterapia no } \\
\text { equilíbrio postural em indivíduo } \\
\text { com sequela de traumatismo } \\
\text { crânio encefálico- estudo de caso }\end{array}$ & Brasil & Relato de caso & $\begin{array}{l}\text { Houve melhora significativa na no equilíbrio } \\
\text { e coordenação motora na deambulação, após } \\
\text { a prática da equoterapia }\end{array}$ \\
\hline $\begin{array}{l}\text { Mutoh, T } \\
\text { et al } \\
(2018)\end{array}$ & $\begin{array}{l}\text { Impact of serial gait analyses on } \\
\text { long-term outcome of } \\
\text { hippotherapy in children and } \\
\text { adolescents with cerebral palsy. }\end{array}$ & Japão & Estudo prospectivo & $\begin{array}{l}\text { Aumento da função motora grossa, } \\
\text { comprimento da passada, a velocidade da } \\
\text { caminhada. }\end{array}$ \\
\hline $\begin{array}{l}\text { Hsieh YL } \\
\text { et al (2017) }\end{array}$ & $\begin{array}{l}\text { Effects of hippotherapy on body } \\
\text { functions, activities and } \\
\text { participation in children with } \\
\text { cerebral palsy based on ICF-CY } \\
\text { assessments. }\end{array}$ & Taiwan & Ensaio clínico & $\begin{array}{l}\text { Melhora nas funções neuro- } \\
\text { musculoesqueléticas e na execução de } \\
\text { atividades e envolvimento social. }\end{array}$ \\
\hline $\begin{array}{l}\text { Matusiak- } \\
\text { Wieczorek } \\
\text { E, et al } \\
\text { (2016) }\end{array}$ & $\begin{array}{l}\text { Influence of Hippotherapy on } \\
\text { Body Balance in the Sitting } \\
\text { Position Among Children with } \\
\text { Cerebral Palsy. }\end{array}$ & Polônia & Estudo comparativo & $\begin{array}{l}\text { Melhora no movimento dos membros } \\
\text { superiores, o qual possibilitou melhor postura } \\
\text { e capacidade de manter o equilíbrio na } \\
\text { posição sentada. }\end{array}$ \\
\hline $\begin{array}{l}\text { Vargas LPJ } \\
\text { et al (2016) }\end{array}$ & $\begin{array}{l}\text { Aplicación de la hipoterapia en } \\
\text { los niños con parálisis cerebral. }\end{array}$ & Espanha & Estudo retrospectivo & $\begin{array}{l}\text { Se mostrou eficaz no desenvolvimento de } \\
\text { habilidades, principalmente no equilíbrio, } \\
\text { atenção e concentração da criança. }\end{array}$ \\
\hline $\begin{array}{l}\text { Kwon JY } \\
\quad \text { et al } \\
(2015)\end{array}$ & $\begin{array}{l}\text { Effect of hippotherapy on gross } \\
\text { motor function in children with } \\
\text { cerebral palsy: a randomized } \\
\text { controlled trial }\end{array}$ & Coréia & Estudo randomizado & $\begin{array}{l}\text { A hipoterapia afeta positivamente o } \\
\text { desempenho e equilíbrio de crianças com } \\
\text { paralisia cerebral }\end{array}$ \\
\hline $\begin{array}{l}\text { Manikowsk } \\
\text { a F et al } \\
(2013)\end{array}$ & $\begin{array}{l}\text { The effect of a hippotherapy } \\
\text { session on spatiotemporal } \\
\text { parameters of gait in children } \\
\text { with cerebral palsy - pilot study }\end{array}$ & Polônia & Estudo piloto & $\begin{array}{l}\text { Os efeitos da hipoterapia, na velocidade da } \\
\text { marcha foram estatisticamente significativas, } \\
\text { portanto, sobre os parâmetros espaço- } \\
\text { temporais, a terapia mostrou-se benéfico para } \\
\text { crianças com PC. }\end{array}$ \\
\hline $\begin{array}{c}\text { Silkwood- } \\
\text { sherer DJ et } \\
\text { al } \\
(2012) \\
\end{array}$ & $\begin{array}{l}\text { Hippotherapy - an intervention to } \\
\text { habilitate balance deficits in } \\
\text { children with movement } \\
\text { disorders: a clinical trial }\end{array}$ & EUA & Estudo de coorte & $\begin{array}{l}\text { Redução dos déficits de equilíbrio e melhora } \\
\text { no desempenho das habilidades de vida diária }\end{array}$ \\
\hline $\begin{array}{c}\text { Frank A } \\
\text { et al (2011) }\end{array}$ & $\begin{array}{l}\text { Effect of hippotherapy on } \\
\text { perceived self-competence and } \\
\text { participation in a child with } \\
\text { cerebral palsy. }\end{array}$ & EUA & Relato de caso & $\begin{array}{l}\text { Melhora na qualidade de vida, aquisição de } \\
\text { competências, aumento no desempenho em } \\
\text { atividades cotidianas, bem como na aceitação } \\
\text { social, }\end{array}$ \\
\hline $\begin{array}{l}\text { Kwon JY } \\
\text { et al (2011) }\end{array}$ & $\begin{array}{l}\text { Effects of Hippotherapy on Gait } \\
\text { Parameters in Children with } \\
\text { Bilateral Spastic Cerebral Palsy }\end{array}$ & Coréia & Estudo randomizado & $\begin{array}{l}\text { Melhora significativa na velocidade da } \\
\text { marcha, comprimento da passada e inclinação } \\
\text { pélvica. }\end{array}$ \\
\hline $\begin{array}{l}\text { Araújo } \\
\text { EAR, et al } \\
(2010)\end{array}$ & $\begin{array}{l}\text { The hippotherapy in the treatment } \\
\text { of children with cerebral palsy in } \\
\text { the North East Brazil }\end{array}$ & Brasil & Estudo descritivo & $\begin{array}{l}\text { Ganhos expressivos na simetria da postura, } \\
\text { coordenação dos movimentos, tonicidade } \\
\text { habilidades motoras e autoestima e } \\
\text { socialização }\end{array}$ \\
\hline
\end{tabular}

Fonte: Autores. 
Após a leitura dos artigos selecionados, emergiram duas categorias, descritas a seguir:

\section{Melhora nas Habilidades Motoras das Crianças com PC}

A terapia com animais, mais especificamente com equinos, vem ampliando-se consideravelmente, especialmente em crianças com paralisia cerebral, pois entende-se que essa modalidade, possibilita uma melhora significativa na função motora.

Pesquisadores, evidenciaram benefícios significativos na função motora grossa (Mutoh et al. 2018; Park et al, 2014; Know et al, 2015) e no equilíbrio (Vargas et al,.2016; Silkwood-Sherer et al,.2012) em vários níveis funcionais, otimizando, quando associado a outra modalidade de fisioterapia tradicional.

Destarte, os estudos supracitados, consideram a terapia assistida por cavalos, como método importante para o equilíbrio e coordenação de movimentos reflexos, além de induzir a criança a um padrão de marcha. As mudanças imediatas no padrão de marcha de crianças com paralisia cerebral, tem chamado a atenção dos pesquisadores, com avanços favoráveis na marcha simétrica e comprimento do passo (Know et al, 2011; Araujo \& Fonseca, 2010).

Melhoras na psicomotricidade, controle de tronco e movimentos da cabeça, foi observado nos estudos como benéficos, após adotar a terapêutica, assim como o desempenho funcional de habilidades da vida diária em crianças com leve e moderados problemas motores (Manikowska et al, 2013; Matusiak-Wieczorek et al,.2016; Hsieh et al,.2017; Frank \& Dole, 2011). No entanto, apesar dos benefícios evidenciados nesses estudos, foram sugeridas que novas pesquisas, preferencialmente randomizadas e com número maior de pacientes fossem realizadas.

Conforme o animal se desloca, vão ocorrendo vários ajustes no corpo da pessoa montada, na qual esses ajustes estimulam músculos que o montador usaria para andar e, ao mesmo tempo a pessoa se esforça para manter equilíbrio.

\section{Qualidade de Vida da Criança com PC}

Outro aspecto a ser discutido é os benefícios da equoterapia na qualidade de vida das crianças com paralisia cerebral. Frequentemente, crianças com PC são isoladas socialmente, devido o desconhecimento da população frente a doença. Somado a isso, a falta de acesso aos locais públicos e espaços de lazer corroboram para este isolamento. Tão importante como a função motora, a equoterapia possibilita, uma melhora nas habilidades cognitivas, e nas relações interpessoais, pelo contato com outras pessoas (Silkwood-Sherer et al, 2012; Araujo \& Fonseca, 2010; Manikowska et al,.2013; Frank \& Dole, 2011.

A inclusão social foi identificada como relevante, promovendo maior qualidade de vida nas crianças com PC. O aumento na autoconfiança da criança, melhoras significativas no autocuidado, motivação em participar das atividades em grupo e o mais importante, o enfrentamento da doença e adesão ao tratamento foram identificadas nos estudos (Manikowska et al, 2013; Hsieh et al,.2017; Frank \& Dole, 2011).

O efeito positivo da terapia assistida por cavalos, encontrados na literatura, através dos artigos selecionados, apontam a importância do estudo não somente a curto prazo, mas ao longo do ciclo vital. A melhora no desempenho na vida diária, tem mostrado o quanto é indicada a terapia com animais no tratamento auxiliar da paralisia cerebral, haja vista uma série de aspectos que perpassam no cotidiano das crianças e das famílias que convivem com o preconceito e a discriminação.

Para as crianças, as frustações, a carência afetiva, a noção de espaço e a consciência das limitações, confirmam a necessidade de os profissionais conhecerem a doença, bem como alternativas que possibilite um cuidado humanizado.

\section{Limitações do Estudo}

Como limitações, indica-se o número escasso de estudos encontrados na literatura, principalmente estudos brasileiros, o que limita a discussão dos benefícios aplicados a realidade nacional. Embora poucos estudos realizados no Brasil, sabe-se 
que o uso dessa modalidade é frequente no Brasil, inclusive relatos em sites e blogs, ressaltando os resultados satisfatórios frente aos benefícios da terapia.

Outra limitação do estudo é a ausência de outras categorias profissionais no atendimento a essas crianças. A maioria dos estudos encontrados, são realizados com profissionais fisioterapeutas, sendo que, de acordo a recomendação da equoterapia, a atividade pode ser exercida por uma equipe multiprofissional. Assim, dentre as dificuldades e limitações encontradas neste estudo, foi a carência de trabalhos que enfatizem a atuação do profissional de saúde, principalmente estudos primários.

No entanto, os resultados tornam o estudo contributivo para o repensar das práticas profissionais, e para a pesquisa na área da saúde, principalmente no Brasil, haja vista um projeto de lei que regulamenta o uso da equoterapia em pessoas com necessidades especiais.

Nesse sentido, constata-se a necessidade de mais estudos realizados no contexto brasileiro, bem como a inserção de outras categorias profissionais no tratamento a crianças com paralisia cerebral. Ademais, se faz necessário, aprofundar o conhecimento dos profissionais que atuam na área de reabilitação e cuidado de crianças com necessidades especiais, visto que essa é apenas uma das terapias inovadoras que podem beneficiar esses pacientes.

\section{Implicações Futuras e Conclusões}

A produção de conhecimento nesta área pode contribuir para que os profissionais de saúde, atue na promoção de saúde e na prevenção de agravos, através de cuidados e orientações individuais, assim como propor intervenções que visem melhorar a qualidade de vida dos pacientes e de suas famílias, encontrando subsídios para a melhoria das práticas assistenciais.

A paralisia cerebral, ao se destacar como uma doença que afeta a saúde e a qualidade de vida, se constituiu como um tema importante para saúde. A terapia com cavalos, embora seja uma modalidade aparentemente atrelado a fisioterapeutas, médicos veterinários, e demais categorias profissionais podem contribuir em um melhor aproveitamento da utilização de estratégias de intervenção em crianças com paralisia cerebral.

A aprovação recentemente da Lei Federal, abre caminhos, não somente para as pessoas que irão se beneficiar da terapia, mas também para os profissionais que poderão se capacitar e ter mais um campo de atuação. Aos pesquisadores, estudos poderão ser realizados, corroborando com a literatura já existente, confirmando a sua eficácia.

Embora uma amostra pequena, esses estudos representam uma importante contribuição do conhecimento a área da saúde, tendo em vista uma modalidade não convencional de fisioterapia para crianças com PC, que se mostrou, através da literatura, melhora significativa no desenvolvimento biopsicossocial da criança com paralisia cerebral.

A indicação da equoterapia complementar a terapia convencional, a partir dos resultados apresentados, é favorável ao identificá-la como uma modalidade terapêutica que tem contribuído para melhor desempenho da coordenação motora e aspectos psicossociais, tão importantes para o desenvolvimento neurológico e psicomotor da criança.

\section{Referências}

Araujo, A. E. R., Silva, V. S, \& Fonseca, B. T. (2010). The hippotherapy in the treatment of children with cerebral palsy in the North East Brazil. Fisioter. Bras 11(1):4-8. http://www.portalatlanticaeditora.com.br/index.php/fisioterapiabrasil/article/viewFile/1323/2446

Associação Brasileira de Paralisia Cerebral (ABPC). (2012). Definição e classificação. http://paralisiacerebral.org.br.

Brasil. (2019). Lei $\mathrm{n}^{\circ}$ 13.830, de 13 de maio de 2019 que dispõe sobre a prática da equoterapia. https://legis.senado.leg.br/norma/3 0940789/publicacao/30940896

Brasil. (2005). Congresso Nacional. Lei no 5.499, de 5 de junho de 2005. Acrescenta parágrafo único ao Art. 18 da Lei no 7.853 , de 24 de outubro de 1989 , que dispõe sobre o apoio às pessoas portadoras de deficiência, para tornar disponível a equoterapia no âmbito do Sistema Único de saúde. Brasília DF.

Cooper, H. M. (1982).The integrative research review: a systematic approach. Sage Publications. 
Frank, A., McCloskey, S., \& Dole, R. L. (2011). Effect of hippotherapy on perceived self-competence and participation in a child with cerebral palsy. Pediatr Phys Ther.,23(3): 301-8. http://dx.doi.org/10.1097/PEP.0b013e318227

Graham, H. K., Rosenbaum, P., Paneth, N., Dan, B., Lin, J. P., Damiano, D. L., et al. (2016). Cerebral palsy. Nat Rev Dis Primers. 2:15082

Hidecker, M. J. C., Slaughter, J., Abeysekara, P., Ho. N. T, Dodge, N., et al. (2018).Early Predictors and Correlates of Communication Function in Children with Cerebral Palsy. Journal of Child Neurology, 33(4): 255-59. https://doi.org/10.1177/0883073817754006.

Hsieh, Y. L., Yang, C. C, Sun, S. H, Chan, S. Y, Wang, T. H, \& Luo, H. J. (2017).Effects of hippotherapy on body functions, activities and participation in children with cerebral palsy based on ICF-CY assessments. Disability and Rehabilitation; 39 (17):1703-1713. http://dx.doi.org/10.1080/09638288.2016.1207108

Know, J. Y., Chang, H. J., YI, S. H., Lee, J. Y., Shin, H. Y, \& Kim, Y. H. (2015). Effect of hippotherapy on gross motor function in children with cerebral palsy: a randomized controlled trial. J Altern Complement Med.;21(1):15-21.

Kwon, J. Y., Chang, H. J., Lee, J. Y, Ha, Y, Lee, P. K., \& Kim, Y. H.(2011). Effects of hippotherapy on gait parameters in children with bilateral spastic cerebral palsy. Arch Phys Med Rehabil. 92(5): 774-9. http://dx.doi.org/10.1016/j.apmr.2010.11.031

Manikowska, F., Jóźwiak, M., Idzior, M., Che,n P. J., \& Tarnowsky D. (2013). The effect of a hippotherapy session on spatiotemporal parameters of gait in children with cerebral palsy - pilot study. Ortopedia Traumatologia Rehabilitacja. 15(6): 253-257. http://dx.doi.org/10.5604/15093492.1058420.

Matusiak-Wieczorek,E, Małachowska-Sobieska, M., \& Synder M. (2016). Influence of Hippotherapy on Body Balance in the Sitting Position Among Children with Cerebral Palsy.Ortop Traumatol Rehabil. 23(2): 165-175. http://dx.doi.org/10.5604/15093492.1205024.

Meincke, N. M., Mélo, T. R., Bonamigo, E. C. B., \& Strassburger, S. Z. (2018). Funcionalidade em atividades de vida diária de crianças deambuladoras com paralisia cerebral. Saúde. 44(3):1-10. http://dx.doi.org/ 10.5902/2236583434885.

Mutoh, T., Mutoh, T., Tsubone, H., Takada, M., Doumura, M, Ihara M, et al. (2018).Impact of serial gait analyses on long-term outcome of hippotherapy in children and adolescents with cerebral palsy. Complement Ther Clin Pract. 30: 19-23. http://dx.doi.org/10.1016/j.ctcp.2017.11.003

Park, S. K, Yang D. J, Heo J. W, Kim J. H, Park S. H, \& Uhm Y. H. (2016).Study on the quality of life of children with cerebral palsy. J Phys Ther Sci; 28(11):3145-48. http://dx.doi.org/10.1589/jpts.28.3145

Park, E. S, Rha, D. W, Shin, J. S., Kim, S., \& Jung S. (2014) Effects of hippotherapy on gross motor function and functional performance of children with cerebral palsy. Yonsei Medical Journal. 55(6): 1736- 1742. http://dx.doi.org/10.3349/ymj.2014.55.6.1736

Prieto, A. V, Silva, F. C, Silva, R, Santos J. A. T, \& Filho, P. J. B. G. (2018). A equoterapia na reabilitação de indivíduos com paralisia cerebral: uma revisão sistemática de ensaios clínicos. Cad. Bras. Ter. Ocup.; 26(1):207-218. http://dx.doi.org/10.4322/2526-8910.ctoAR1067

Silkwood-sherer D. J, Killian C. B, Long T. M, \& Martin K. S. (2012). Hippotherapy - an intervention to habilitate balance deficits in children with movement disorders: a clinical trial. Physical Therapy; 92(5): 707-717. http://dx.doi.org/10.2522/ptj.20110081

Shikako-Thomas K, Shevell M, Schmitz N, et al. (2013). QUALA Group: Determinants of participation in leisure activities among adolescents with cerebral palsy. Res Dev Disabil, 34: 2621-2634. http://dx.doi.org/10.1016/j.ridd.2013.05.013

Vargas L. P. J, Cartas U. S, Larrarte J. P. M, \& Espinosa I. S. (20160. Aplicación de la hipoterapia en los niños con parálisis cerebral. Rev. Arch Med Camagüey. 20(5): 496-506. http://scielo.sld.cu/pdf/amc/v20n5/amc060516.pdf. Acesso em 15/05/2019. 